\title{
Hepatitis b virus (hbv) and s-escape mutants: from the beginning until now
}

\begin{abstract}
In spite of the progress made in vaccine and antiviral therapy development, hepatitis B virus (HBV) infection remains a major health care problem. More than 240 million people are chronically infected worldwide showing differences in the severity of liver disease, clinical outcome and response to immune- and antiviral-therapy. Parameters associated to the host immune system (HBV specific T- and/or B-cell repertoires, defective antigen presentation and diminished Th1/Th2 response ratio) and viral factors such as the HBV genotypes and their evolving variants/mutants, have largely contributed to the explanation of such differences. The unique genomic structure and replication cycle of HBV provide much opportunity for mutations to occur in any of its genes undergoing selection pressures, such as those associated with the host immune system, the hepatitis B vaccine and/or hepatitis B immune globulin and the antiviral therapy with nucleos(t)ide analogues. First, this review describes the current prevalence of S-escape mutants worldwide. Then, the clinical implications of such surface gene variants and the impact of universal hepatitis $B$ vaccination on HBV mutations and genotypes are discussed. Finally, the fact that the immune escape process also extends well beyond HBV are addressed.
\end{abstract}

Keywords: HBV, HBsAg, S-immune escape mutants, Hepatitis B vaccine, OBI, HBIG
Volume 2 Issue 3 - 2015

\author{
Priscila Perazzo,' Nair Eguibar,' Rodrigo H \\ Gonz lez, ${ }^{2}$ Alejandro D Nusblat, ${ }^{2}$ Mar a L \\ Cuestas' \\ 'Institute of Microbiology and Medical Parasitology, University \\ of Buenos Aires-National Science Research Council (IMPaM, \\ UBA-CONICET), Argentina \\ ${ }^{2}$ Catedra de Biotecnologia y Microbiologia Industrial, \\ Universidad de Buenos Aires, Argentina
}

\begin{abstract}
Correspondence: Maria L Cuestas, Institute of Microbiology and Medical Parasitology, University of Buenos Aires-National Science Research Council (IMPaM, UBACONICET), 2155 Paraguay St, I I th Floor, CI I I ABG, BuenosAires, Argentina, Tel +54 II 5950 9500, Fax 54 II 4964 2554; Emailmarilucuestas@gmail.com
\end{abstract}

Received: February 19, 2015 | Published: April 18, 2015
Abbreviations: WHO, World Health Organization; HBV, Hepatitis B Virus; CHB, Chronic Hepatitis B; HCC, Hepatocellular Carcinoma; HIV, Human Immunodeficiency Virus; ART, Antiretroviral Therapy; OBI, Occult HBV Infection; HDV, Hepatitis Delta Virus; HBIG, Hepatitis B Immune Globulin; ORF, Open Reading Frame; aa, Amino Acids; HBsAg, Hepatitis B Surface Antigen; cccDNA, Covalently Closed Circular DNA; MHR, Major Hydrophilic Region; MHC, Major Histocompatibility Complex Class; OBH, Occult Hepatitis B

\section{Introduction}

In accordance with data provided by the World Health Organization (WHO), hepatitis B virus (HBV) chronically infects almost 240 million people worldwide despite the availability of the hepatitis $\mathrm{B}$ vaccine since 1982. ${ }^{1}$ Chronic hepatitis B (CHB) is an important global health concern due to its significant morbidity and mortality. It is the cause of approximately $50 \%$ of the world's cases of hepatocellular carcinoma (HCC) and about $30 \%$ of all cases of liver cirrhosis. ${ }^{2}$ leading to over 780,000 annual deaths. ${ }^{3}$

In the last few years, considerable progress has been made in the construction and development of effective hepatitis B vaccines and in the implementation of childhood immunization programs so as to protect future generations. In this regard, in 1992 the WHO passed a resolution which recommended universal infant vaccination against hepatitis B. As a result, 183 countries around the world have incorporated the hepatitis $\mathrm{B}$ vaccine into their corresponding national childhood immunization schedules. ${ }^{1}$ However, several gaps remain to be solved. Firstly, the implementation of hepatitis B vaccination is still considerably low where it is most needed, especially in Central Africa, where the prevalence rates of $\mathrm{HBV}$ infection are the highest to be reported. ${ }^{4,5}$ Secondly, some of the vaccinated infants still sometimes get infected due to vaccine-escape mutants. ${ }^{6-9}$

Thirstily, in the era of dual active antiretroviral therapy (ART) used in HBV/human immunodeficiency virus (HIV) co-infected patients ART-induced vaccine-escape HBV mutants emerged as a new hazard. In this case, vaccine-escape HBV mutants arises not from attempts to escape from selection resulting from host immune surveillance or exposure to hepatitis B Immuno prophylaxis, but from treatment-induced mutations from the overlapping genes (i.e. viral polymerase mutations induce both drug resistance and vaccine-escape $\mathrm{S}$ gene mutants). ${ }^{10}$ Lastly, vaccine-escape mutants may also cause occult HBV infection (OBI), misleading to false negative results in diagnosis (diagnostic-escape mutants), a problem that may extend also to hepatitis delta virus (HDV)/HBV co-infected patients, since the first step in the diagnosis of HDV infection is testing HBsAgpositive individuals for the antibody to the HD antigen . ${ }^{11}$

Consequently, HBV vaccine-escape mutants still constitute a matter of public health concern.

In this manuscript, the terms HBV vaccine-escape mutants, hepatitis B immune globulin (HBIG)-escape mutants, HBV immuneescape mutants and S-mutants will be used indistinctly. Firstly, the present review describes the current prevalence of S-escape mutants around the world and their clinical implications. Their impact on diagnosis, universal hepatitis $\mathrm{B}$ vaccination, and the effectiveness of the current genotype-A vaccine to induce cross protection against the other genotypes are discussed. Finally, some fresh insights into the growing problem of HBV immune- and/or therapy-escape variants and the finding of an increasing detection of these variants in HIV co-infected patients are addressed.

\section{The structure of hepatitis B virus}

HBV is a species of the genus Orthohepadnavirus that is part of the Hepadnaviridae family, whose genome consists of a 3.2-kb-long partially double-stranded relaxed-circular DNA molecule containing four overlapping open reading frames (ORFs): the surface (S-), the core (C-), the polymerase (P-) and the X- genes codifying for all viral proteins. ${ }^{12}$ The S-ORF includes the sequence assumed to codify the binding sites for the hepatocyte and has three in-frame AUG codons 
dividing this ORF into three regions, known as Pre-S1, Pre-S2, and $\mathrm{S}$, encoding for these homonym viral envelope proteins. ${ }^{13}$ The C-ORF has two initiation codons encoding for the structural protein of the nucleocapsid, which plays an important role in viral particle assembly (hepatitis B core antigen $[\mathrm{HBcAg}]$ ), and a soluble antigen secreted by infected cells (hepatitis $\mathrm{B}$ e antigen $[\mathrm{HBeAg}]$ ). ${ }^{14}$ The X-ORF encodes for the hepatitis $\mathrm{B} \times$ antigen [HBxAg], which displays a Trans activating activity and other regulatory functions. ${ }^{15}$ Finally, the P-ORF encodes for a multifunctional enzyme, the polymerase, which constitutes the current target for the nucleos(t)ide analogues used in the treatment of CHB. ${ }^{14}$

The lack of proof-reading activity of HBV polymerase results in a high mutation rate of 1.4 to $3.2 \times 10-5$ nucleotide substitutions per site per year. ${ }^{16}$ This figure is one to two orders of magnitude lower than in other viruses lacking polymerase-associated proof-reading functions, such as RNA viruses. ${ }^{17}$ and four orders of magnitude greater than in "common" (without reverse transcriptase) DNA viruses. ${ }^{18}$ Therefore, HBV populations may evolve at a faster rate than most DNA viruses as a result of environmental factors; hence, viral HBV mutants may likely emerge in response to those factors. Lately, an estimated rate of $7.9 \times 10-5$ nucleotide substitutions per site per year has been recorded after analyzing the evolution of 8 full-length genotype B DNA genomes from untreated patients (HBeAg minus) during a 25 -yr period. ${ }^{19}$ It is interesting to note that a rate of $2.7 \times 10-3$ nucleotide substitutions per site per year was recorded when such rate was studied during a 3-yr period in a patient infected with an $\mathrm{HBeAg}$ minus genotype $\mathrm{F}$ strain in the presence of anti-HBs antibodies -that is, with $\mathrm{HBeAg}$ and HBsAg mutants. ${ }^{20,21}$

Additionally to the well known T-cell epitopes found within the $\mathrm{C}$ and $\mathrm{S}$ proteins, there were also described highly conserved amino acidic sequences within the viral polymerase, which are CD8+/CD4+ T-cell epitopes involved in viral clearance and even -in some casesin the development of subclinical forms of hepatitis B disease. ${ }^{22}$ Therefore, there is a seeming paradox in that natural infection progresses efficiently in spite of the production of highly immunogenic particles that are able to elicit neutralizing host responses.

\section{HBsAg}

Pre-S1, Pre-S2 and S, the three HBV envelope proteins, are translated from specific mRNA. The $S$ protein, which is coded for the $\mathrm{S}$ gene, is made up of 226 amino acids (aa) and is the main component of the viral envelope. ${ }^{23}$ The Pre-S2 protein codes for the Pre-S2 region ( $55 \mathrm{aa}$ ) and the $\mathrm{S}$ gene, whereas the Pre-S1 protein codes for the Pre-S1 region (108 or 119 aa, depending on genotypes), the Pre-S2 region, and the $\mathrm{S}$ gene. Therefore, all these three envelope proteins sharing the same carboxy-terminal end or hepatitis B surface antigen ( $\mathrm{HBsAg}) .^{24}$ can be easily detected by regular immunoassays for diagnosing both HBV and HDV infections and screening of blood donors around the world. Pre-S1 and possibly the Pre-S2 protein would likely mediate binding and entry of the virus into the hepatocytes. The Pre-S1 protein also regulates the nuclear pool of covalently closed circular DNA (cccDNA).

Finally, all three envelope proteins -Pre-S1, Pre-S2, and Sparticipate in generating and secreting the enveloped virus. To date, the pleomorphic nature of HBsAg particles has not allowed their crystallization; therefore, their precise three-dimensional structure has not been elucidated yet. Nevertheless, several models combining data related to their hydrophobicity, hydrophobic moments, flexibility, secondary structure prediction and antigenicity were suggested to determine the putative structure of these HBsAg-containing particles. ${ }^{25-28}$ However, it is now widely accepted that their antigenicity mainly depends on the spatial structure of the $a$ determinant within the major hydrophilic region (MHR), which comprises aa 101 to 160 of the $\mathrm{S}$ protein and is exposed on the surface of both virions and sub viral particles. This region is characterized by high immunogenicity and is potentially under selective pressure of the immune system. ${ }^{24}$

The $a$ determinant is a complex conformational region and disulfide bridges among highly conserved cystein-residues play a significant role in the preservation of the native tertiary structure of this important HBsAg determinant. ${ }^{24,25,27}$ Of the 14 cysteinresidues in the 226-aa-sequence common to all three HBV envelope proteins, 8 are found within the MHR of HBsAg, being important in preserving the structural conformation and therefore the antigenicity of the $a$ determinant. ${ }^{25,27}$ It is thought that the $a$ determinant is made up of two loops bound together by disulfide bridges between $\mathrm{C} 107$ and C138, and between C139 and C147. ${ }^{25,27,28}$ This important determinant is considered the main neutralization epitope and is common to all HBV genotypes. Thus, antibodies targeting this determinant are supposed to protect against re-infection with any of the HBV genotypes. ${ }^{29}$ Changes in the conformation which can successively alter the binding of neutralizing antibodies can occur due to amino acid substitutions within the $a$ determinant. ${ }^{30}$

\section{HBsAg mutants}

A HBV mutant is defined as a variant that arises as a result of specific selection, conferring a specific phenotype. ${ }^{31}$ In the case of vaccine-escape mutants this specific selection is conferred by forces such as the host immune system that leads to mutations in the S-ORF or the antiviral therapy that leads to mutations in the overlapped P-ORF. HBV mutants remain stable over time and their transmission can occur horizontally or vertically. ${ }^{29}$ Carman et al propose that forces such as the humoral and cellular immune response will select some variants from the HBV pool having similar replication potentials; these variants, are known as immune escape mutants, as for example the HBsAg immune-escape mutants. ${ }^{28}$ Therefore, the resulting mutant viruses are allowed to escape from both the humoral and cellular host's immune response by mutated S-genes, thus reducing diagnostic and Immuno prophylaxis efficacy. Many of these S-immune-escape mutants, such as G145R and D144A, can cause HBV-infections in individuals who have been successfully vaccinated. ${ }^{32}$

HBsAg-immune-escape mutants may be found both within and without the $a$ determinant. ${ }^{30}$ In this connection, it was suggested that areas located upstream and downstream of the MHR may play an important role in neutralization. ${ }^{33}$ Mutations occurring outside the MHR are frequently seen, having a tendency to cluster in two regions: around codons 44 to 49 and 152 to 213 . The first region contains both a major histocompatibility complex class I (MHC-I)-restricted T-cell epitope and a B-cell epitope, while the second region, at least up to aa 207, displays both MHC-II T helper epitopes and B-cell epitopes. It was also reported that changes within this second region, located immediately downstream of the $a$ determinant, may cause alterations in the structural component of such immunogenic determinant. ${ }^{30,34}$

In accord with this notion, many researchers have demonstrated that the binding to anti-HBs antibodies is annulled by aa insertions and deletions in this region. ${ }^{35,36}$ Consequently, HBsAg mutations may occur in aa substitutions, insertions, and/or deletions. Ogura et al. noted that during the natural course of infection a high proportion of mutations within the $a$ determinant arise within the first loop (aa 107 to 138), whereas those mutations induced under immune pressure as a result of active and/or passive immunization most frequently occur within the second loop (aa 139 to 147). ${ }^{37}$ 


\section{Discussion}

\section{Current prevalence of S-escape mutants}

HBsAg escape mutants may develop in HBV infected individuals after vaccination with hepatitis B vaccine and/or HBIG administration, in chronic HBV patients during the natural course of HBV infection, and/or in chronic HBV patients receiving antiviral therapy with nucleos(t)ide analogues as a result of the selective pressure exerted by the host immune system or by the antiviral drugs currently used as therapy. These selective pressures allow the survival of the fittest variant. $^{38}$

The first report mentioning the emergence of vaccine-escape mutants dates back to 1988 in an Italian boy ${ }^{39}$ Since then, these mutants have been reported by several countries worldwide. Among them, G145R is considered to be the most relevant and best-documented mutation as well as the most commonly found. Such mutant is also stable over time, can replicate to high titers over long periods of time and can be horizontally transmitted. ${ }^{40}$

Other HBsAg-immune-escape mutants that have been reported throughout the world are those having aa substitutions at positions $120,123,124,126,129,131,133,141$ and 144 of the $a$ determinant, insertions of 1, 2, 3 and 8 aa..$^{29,41,42}$ and deletions..$^{43}$ Other reported mutations abolishing the two-loop structure of the $a$ determinant and producing changes in the hydrophobicity, the electrical charge or the acidity of the loops are those at positions 127, 130, 134, 142 and 146. Moreover, these alterations in the two-loop structure could be explained by the presence of possible additional N-linked glycosylation sites (G130N, for example) or a change in disulfide bridge stability (for example, C147G). ${ }^{41}$ S-escape mutants have also been detected at positions 159, 183 and 184, outside the $a$ determinant and -even- outside the MHR.

These mutants can be found both in vaccinated or unvaccinated individuals and may also fail to bind properly to anti-HBs antibodies in many commercial diagnostic kits used for HBsAg detection during hepatitis B disease screening, and also during hepatitis D screening. ${ }^{29,42}$ Mutations that only change the subtype alleles $w$ to $r(\mathrm{~K}$ 160R) but do not modify the $a$ determinant are not clinically relevant. Nevertheless, there could be a correlation between the subtype allelic mutation $d$ to $y(\mathrm{~K} 122 \mathrm{R})$ and a higher failure rate of passiveactive Immuno prophylaxis in infants of $\mathrm{HBeAg}$ positive mothers. ${ }^{44}$ Mutations between codons 40 and 49, and between codons 198 and 208 not altering the $a$ determinant were identified in patients having HBIG prophylaxis following orthotropic liver transplantation. ${ }^{45}$ Those mutations occurring within the first region could be selected by the immune pressure since this region bears both a MHC-I-restricted T-cell epitope and a B-cell epitope. On the other hand, the second region displays both MHC-II T helper epitopes and B-cell epitopes. ${ }^{41}$

Ma \& Wang assessed 11,221 non-redundant HBV sequences of 8 genotypes (from A to $\mathrm{H}$ ), recovered from the National Center for Biotechnology Information (NCBI) and established the prevalence of S-immune escape mutants in the different HBV genotypes. ${ }^{46}$ On the one hand, they reported the prevalence of S-immune escape mutants P120T, T126S, Q129H, G130N, S143L, D144A and G145A/R, which are related to diagnostic failure in 1 or more genotypes, exhibiting a frequency of no less than $1 \%$. On the other hand, they reported the prevalence of S-escape mutants associated with vaccine or HBIG failure at positions 120,126, 129, 130, 133, 134, 137, 140, 143, 144 and 145; however, they observed great heterogeneity among the different HBV genotypes. ${ }^{46} \mathrm{~A}$ cross-sectional study carried out in adult patients with liver disease in India, showed that the prevalence of
S-immune escape mutants was $0.7 \%{ }^{47}$ In Italy, the prevalence of the G145R mutant was 3.1\%. In that study, authors reported that in $62.5 \%$ of cases, the G145R mutant was alone, whereas in $37.5 \%$ of cases, it was accompanied by multiple mutations (T126I-T131A-C139Y-E/ D144G, T126I-M133L, and P120Q-T126I). ${ }^{48}$

In a large study carried out in Singapore, about $12 \%$ of the infants born to carrier mothers $\mathrm{HBsAg}[+]$ and $\mathrm{HBeAg}[+]$ were infected with HBV despite receiving hepatitis B vaccination and HBIG at birth. ${ }^{49}$

The authors of a research paper from China reported that there was a $3.4 \%$ failure rate in the HBV vaccine evaluated in the Chinese adult population involved in the HBV vaccination program..$^{50}$ Another study also conducted in China, compared the prevalence of HBV mutants in children and adults between 1992 - the year of nationwide HBV vaccination program implementation in China- and 2005. ${ }^{51}$ The study showed that the prevalence of $S$-immune escape mutants in children increased from $6.5 \%$ in 1992 to $14.8 \%$ in 2005 , where the G145R mutant was found to occur more frequently.

In contrast, there was little difference in mutation frequencies between 1992 (9.4\%) and 2005 (9.9\%) in adults. ${ }^{51}$ In that study, authors concluded that immunization in China increased the HBsAg mutation frequencies and the prevalence of Pre-S1 mutants, particularly disease-related mutants. ${ }^{51}$ Moreover, in a study carried out in Taiwan no increase was reported in the prevalence of HBsAg-immune escape mutants in children and adolescents that had been fully covered by universal infant immunization. ${ }^{52}$ However, when authors compared the prevalence of S-escape mutants between immunized and nonimmunized children, aged 1-4 years old, they observed that more S-mutants were found in the first group ( $31 \% \mathrm{vs} 4 \%$, respectively). ${ }^{52}$ It is interesting to note that there were higher frequencies in the emergence of S-escape mutants in those children immunized with plasma-derived vaccines rather than in those immunized with recombinant vaccines $(0.3 \%$ vs $0.06 \%){ }^{52}$ In this connection, it was previously demonstrated that the levels and durability of the anti-HBs antibodies elicited by the second-generation vaccines or recombinant yeast-derived vaccines are lower than those achieved within the $\mathrm{HBsAg}$ vaccine derived from the plasma of infected individuals (firstgeneration vaccines). ${ }^{53}$

Third-generation vaccines or mammalian cells-derived vaccines display a potential superiority over yeast-derived vaccines in helping to avoid vaccine induced escape mutants. This superiority is mainly related to the lack of Pre-S antigens (Pre-S1 and Pre-S2) that carry neutralizing epitopes, and to the presence of misfolded HBsAg in yeast-derived vaccines, whereas mammalian cells-derived vaccines contain correctly folded HBsAg and the neutralizing epitopes of the Pre-S antigens (Pre-S1 and Pre-S2). ${ }^{54}$ Another difference is that human plasma- and recombinant mammalian cell-derived vaccines are glycosylated, whereas yeast-derived HBsAg is not glycosylated either from Saccharomyces cerevisiae or from the methylotropic yeasts Pichia pastoris and Hansenual polymorpha. ${ }^{55}$

In other parts of the world, as for example in Latin America, the epidemiological information related to HBsAg mutants is scarce or practically non-existent. This kind of data is of vital importance in an increasingly immunized world against HBV due to the fact that HBsAg mutants may become set up and spread within populations that are assumed to be protected, and may not be detected by immunodiagnostic kits. Therefore, epidemiological research works from different parts of the world are really essential for gaining insight into the prevalence and characteristics of HBsAg mutants. Once available, such information will be extremely valuable both for producing new HBV vaccine(s) and designing new immunoassays for 
HBsAg detection as well as for avoiding false negative results when screening for HBsAg in blood donors.

Some reports from Argentina have recently documented the emergence of S-immune escape mutants in spite of the presence of usually protective anti-HBs antibodies. ${ }^{8,20,21,30,34,56,57}$ and of cytotoxic $\mathrm{T}$ lymphocyte-specific clones. ${ }^{30}$ Some of them occurred naturally in HBV-chronically infective patients while others are the result of selected immunological pressures upon vaccination. To date, there have not been reports referring to these mutants in other parts of the world. Among the S-mutants reported in Argentina, were C69Y, C90Y, L110I, T114A, C124R, C124Y, C138R, N129R, C139R, S140T, L158F, A168T, C176R, N178S. ${ }^{34}$ S45A, P46H, L49H, C107R, T125A，M133K，I152F，P153T, Y161S, G185E，A194T,G202R, I213L. ${ }^{30}$ D144A, L209V. ${ }^{8}$

In general terms, the worldwide prevalence of S-immune escape mutants due to the selection pressure exerted by the hepatitis $B$ vaccine is low, not yet constituting a threat to public health that would require the modification of the available hepatitis B immunization programs, but this does not mean that improvement in current hepatitis B vaccines is unnecessary. However, nowadays, a concerning statement is related to P-mutants inducing vaccine-escape $\mathrm{S}$ gene mutants due to treatment induced mutations. The overlapping nature of the $\mathrm{S}$ and the P-ORFs in the HBV genome makes that almost every single mutation may exert an influence on more than one function of the corresponding nucleotide sequence.

Hence, mutations that occur in the S-ORF selected by forces such as the immune system may be translated as mutations in the P-ORF that eventually may result in resistance to the antiviral therapy. Likewise, those mutations occurring in the P-ORF selected by forces such as the antiviral therapy may be translated as mutations in the S-ORF that eventually may produce an S-immune escape mutant. The circulation and transmission of S-escape mutants resistant to the nucleos(t)ide analogues currently used as anti-HBV therapy and vice versa, may pose a significant risk for the community in view that these mutants may potentially infect both naïve and immunized individuals and negatively affect the efficacy of both the antiviral treatment and the vaccination programs. ${ }^{56,58}$

In this regards, it is somehow worrisome the increasing number of reports in the international literature concerning the detection of S-escape mutants in HIV co-infected patients during long-term ART. ${ }^{59,60}$ Three major patterns of mutations in the HBV polymerase associated with lamivudine resistance were described in those HBV/ HIV co-infected patients receiving ART with lamivudine as the soleanti-HBV drug: single rtM204V, double rtL180M+rtM204V and triple $\mathrm{rtV} 173 \mathrm{~L}+\mathrm{rtL} 180 \mathrm{M}+\mathrm{rtM} 204 \mathrm{~V}$. This triple mutant corresponds to a vaccine escape mutant due to the corresponding change in the overlapped S-ORF. ${ }^{61}$ Thus, the use of lamivudine as the only active anti-HBV agent is discouraged. ${ }^{62}$ It has been widely accepted that HIV therapy with nucleos $(\mathrm{t})$ ide analogues active against $\mathrm{HBV}$ improves hepatic outcomes in HIV/HBV co-infected patients, especially when tenofovir is used. ${ }^{62}$ However, drug resistance, cross-resistance with other antiviral drugs and the occasionally selection for $\mathrm{HBV}$ vaccineescape mutants are still the major drawbacks of the nucleos(t)ide analogues currently used in the ART regimen.

During an interesting follow-up study carried out by Lacombe et al. in HBV patients co-infected with HIV, authors observed a high frequency of S-immune escape mutants selected by the nucleos(t) ide analogues used as therapy. ${ }^{63}$ Another study showed that antiHBV drug resistant mutations were detected in $54 \%$ of HBV/HIV co- infected patients receiving dual active ART and $45 \%$ of them had an immune escape mutation. ${ }^{64}$

In summary, the prevalence of S-immune-escape mutants displays a great variability among the different group of patients analyzed: vaccinated infants, chronic patients, liver transplant recipients and HBV/HIV co-infected patients. Thus, S-mutants selected by the immune system are responsible for $0.2-4.6 \%$ of the cases of breakthrough infections in newborns that received the corresponding immune prophylaxis at birth, and up to $40 \%$ of the liver transplant recipients that received HBIG as immune prophylaxis, develop S-escape mutants. ${ }^{29}$ At present, the high and steadily increasing incidence of HBV vaccine-escape mutants among HIV co-infected patients receiving dual ART must be seriously considered and a judicious selection of the antiviral agents and vigilant monitoring of viral mutants during the course of therapy are mandatory.

\section{Pathobiology and clinical implications of S-escape mutants}

The clinical spectra of S-mutants in HBV infected patients range from asymptomatic without any sign of hepatic injury to fulminant hepatitis, chronic hepatitis, fibrosing cholestatic hepatitis, cirrhosis and HCC. ${ }^{29}$ Experimental data and studies conducted in humans have demonstrated that some mutations in the S protein may be cytotoxic to the hepatocytes. According to this, mutations related to viral retention and the corresponding characteristic appearance of ground-glass cells may contribute to a worse prognosis due to a more progressive liver disease. ${ }^{65,66}$ Furthermore, Asahina et al have described some mutations in the HBsAg associated to acute exacerbations of CHB such as the amino acidic substitutions L95W, I126S/T, G130N, T131N, M133T/K y V173L. ${ }^{67}$

Hence, it is of the utmost importance to detect S-mutants in CHB patients so as to identify those patients requiring a preventive and appropriate therapy as well as a closer follow-up strategy for early detection of HCC. ${ }^{66}$ As mentioned above, due to the overlapping structure of HBV genome, it was reported that the lamivudineselected mutants E164D/I195M demonstrated a minimal binding of anti-HBs antibodies while the E164D, M198I, I195M and W196S lamivudine-selected mutants also showed reduced binding to antiHBs antibodies. ${ }^{68}$ This phenotype is currently observed in HIV coinfected patients receiving dual ART at a steadily increasing frequency. Moreover, few cases of reactivation of CHB in absence of HBsAg has been reported in individuals with different Immuno deficiencies and in those with HIV co-infection following discontinuation of ART. ${ }^{60}$

However data on clinical and virological outcomes in HBV and HIV co-infected patients receiving active ART are limited and further studies are needed to understand the complex molecular virology of $\mathrm{HBV}$ during HIV co-infection. Another interesting issue is related to HDV co-infected patients. HDV must either co-infect or super-infect with HBV and requires HBsAg and Pre-S antigens to assemble into a new HDV virion in the infected hepatocytes. ${ }^{69}$ Thus S-immune escape mutants may also represent a problem of concern in the hepatitis D clinical setting since their eventual effect in the pathogenicity of HDV. In this regards, it is widely well known that HDV co-infection is usually associated to a worse prognosis in $\mathrm{CHB}$ patients (more frequent cirrhosis, increased risk of $\mathrm{HCC}$ and/or and fulminant hepatitis). In the hepatitis $\mathrm{C}$ virus ( $\mathrm{HCV}$ ) co-infection setting, the role of S-mutants and liver disease is also uncertain.

Finally, S-escape mutants have become an important clinical and public health concern because they might be associated with OBI (presence of viral DNA with undetectable HBsAg and low viral 
replication). ${ }^{70,71}$ Both anti-HBc and/or anti-HBs positive individuals and individuals negative for all serologic HBV infection may be harboring OBI. ${ }^{70,72}$ The exact mechanism of OBI has still to be elucidated. However, such mechanism may be possibly explained by the intra hepatic persistence of cccDNA under the host's vigorous immune suppression of viral replication and gene expression. ${ }^{71}$ Additional mechanisms not related to the host response were also reported, including:

i. The presence of defective particles or of mutations in transcription control regions within the viral polymerase causing low HBV replication and HBsAg expression

ii. The circulation of S-escape mutants

iii. Hepatitis $\mathrm{C}$ virus (HCV) or hepatitis D virus (HDV) co-infection, since these viruses down-regulate HBV replication, thus reducing HBsAg expression

iv. The abolishment of the HBsAg expression due to a RNA splicing event. $^{72-75}$

Several epidemiological and molecular studies demonstrated that OBI can lead to the development and exacerbation of HBV related diseases, such as liver fibrosis, cirrhosis and HCC..$^{76}$ Different virological characteristics were found on HCC patients with OBI, for example, higher frequencies of amino acidic mutations in Pre-S/S such as the presence of premature stop codons or some nucleotidic substitutions in the enhancer II region such as G1721A. The overall conclusion is that, despite the low viral load, the risk of HCC in OBI patients is high. ${ }^{77}$

Another epidemiology study conducted in Taiwan demonstrated that $10.9 \%$ of the HBsAg sero negative vaccinated children had OBI, and although the most documented mutation G145R was not found, other mutations inside the MHR were recorded (C139S and A159T). ${ }^{78}$ Deletions in the 3' terminus of Pre-S1 lead to loss of immune epitopes and to a reduced secretion of HBsAg. ${ }^{78}$ In Immuno compromised patients, OBI may reactivate causing acute hepatitis. ${ }^{71}$ Although the relation between OBI and the development of chronic liver diseases and $\mathrm{HCC}$ is not well understood, it was reported that OBI contributes to the genesis of HCC directly by its own proto-oncogenic effect and indirectly by causing persistent liver inflammation and fibrosis. ${ }^{71}$

As mentioned above, the presence of co-infections usually influences the natural evolution of each of the infectious agents present by either facilitating their virulence or by competing for their resources. Many studies have focused on the clinical impact of HIV, $\mathrm{HCV}$ and $\mathrm{HDV}$ on the natural evolution of $\mathrm{HBV}$ infection, finding that co-infection carries an increased risk of progressive liver disease and HCC. A persisting risk of repeated flares of hepatitis and progressive liver disease is even more frequent when co-infections are present in patients with OBI. ${ }^{79}$

\section{Diagnostic escape mutants}

Today, in many countries around the world, HBV is still one of the serious infectious risks for the blood transfusion safety. ${ }^{46}$ One of the reasons is the worldwide circulation of the S-immune escape mutants that pose a challenge not only to the hepatitis B prophylactic tools but also to the diagnosis; issues that should also be extended to HDV since its absolute requirement for HBV for its infection of hepatocytes, replication and formation of virions. In this regards, Delfino et al. provided valuable evidence of the presence of HDV infection in OBI patients due to S-escape mutants. ${ }^{80}$ Hence, re-evaluation of the HDV diagnostic algorithms is thus mandatory.
Many current ELISA kits used may be unable to detect properly S-immune escape mutants. ${ }^{42}$ Therefore, they may be potentially transmitted horizontally by blood transfusions of an asyntomatic carrier having no known risk factors concerning HBV infection, rendering a false negative result in $\mathrm{HBsAg}$ serology for harboring an $\mathrm{HBsAg}$ mutant virus that commercial HBsAg kits have failed to detect. $^{72,81}$

The introduction of novel HBsAg assays able to detect these S-gene mutants permanently improves the sensitivity of such assays. Full sensitivity is not guaranteed by polyclonal capture antibodybased assays, despite performing much better than most commercially available double monoclonal (capture and tracer) assays, which employ antibodies directed against the second loop of the $a$ determinant. The major disadvantage of employing polyclonal antibody immunoassays is that their specificity is lower than that of monoclonal assays. Therefore, the use of mixtures of monoclonal antibodies that are able to recognize both wild-type and S-mutants strains is advised for HBsAg screening. With the purpose of reducing failure in the diagnosis associated with HBsAg-negative mutants, tracer and capture antibodies against the Pre-S region were recommended for inclusion in diagnostic immunoassays. Nevertheless, if a mixture of antibodies is used, there may be a decrease in the analytical sensitivity for HBsAg of different genotypes even of wild-type virus. Antibodies directed against the second loop of the $a$ determinant should not be used for immunoassays since they are not able to detect G145R mutants. ${ }^{29}$

Weber also suggested that anti-HBc testing should be used in combination with HBsAg for blood donor screening, particularly in those countries where there is no mandatory HBV DNA detection. ${ }^{29}$ by nucleic acid amplification technology (NAT), such as in many regions of Africa and Latin America. For many years, HBV DNA tests were not very sensitive. Nowadays, new generation DNA detection technologies such as real-time PCR and transcription-based mediated amplification (TMA) allowed a decrease in the lower detection limit $(<5 \mathrm{IU} / \mathrm{mL}$ of HBV DNA), which is particularly important in OBI because DNA levels vary at $-5-10 \mathrm{IU} / \mathrm{mL}$ (range $<10$ to 425 copies/ $\mathrm{mL}) .^{79}$

\section{HBV Genotypes and hepatitis B vaccine}

Phylogenetic analysis based on the comparison of complete human HBV genomes has defined at least nine genotypes (A-H, and J) and 34 sub genotypes ${ }^{82} \mathrm{~A}$ putative genotype I has been proposed. ${ }^{83,84}$ but its existence is still controversial. HBV genotypes have been shown to have a distinct geographic distribution and seem to have differences in biological properties that may account for differences in the clinical outcome of the infection or in the antiviral treatment response. ${ }^{29}$

Genotype E from Africa is the most predominant genotype in Western and Central Africa. Genotype E strains isolated in Europe and their exceptional detection in America have been derived from HBV carriers of African origin, regardless of their country of residence. ${ }^{30,8}$. Noteworthy, genotype E shows a marked genotypic divergence with respect to all genotypes within the $a$ determinant which seems to account for vaccination failure and also appears to be an influencing factor for HBsAg screening by diagnostic assays. ${ }^{86}$ Genotype $F$ is the most predominant genotype in Central and South America and it is recognized as the most divergent of all the genotypes.

It is extremely important to note that the current available hepatitis $\mathrm{B}$ vaccine has the HBsAg genotype A2, subtype adw2, whereas genotype $\mathrm{E}$ isolates have subtype ayw4, and genotypes $\mathrm{F}$ isolates have subtypes adw4q- and/or ayw4. The fact that subtype-epitopes are 
very immunogenic, together with the emergence of S-escape mutants, has raised questions about the effectiveness of the current vaccine in Africa where genotype $\mathrm{E}$ is the most predominant HBV genotype, as well as in Central and South America where F genotype is frequent. ${ }^{8,87}$ Finally, in some Asian countries, such as in Japan and Korea, where genotype $\mathrm{C}$ is the most prevalent genotype, vaccines generated from genotype $\mathrm{C}$ are used. Interestingly, in these areas acute hepatitis $\mathrm{B}$ due to genotype $\mathrm{A} 2$ has increased..$^{87}$ Therefore, further studies are needed, since little is known about whether vaccination against one genotype can induce effective cross protection against the others.

\section{Conclusion}

HBsAg mutants, either naturally occurring or selected through immunological or antiviral therapy pressure are of clinical significance due to the role they play in active and/or passive HBV Immuno prophylaxis failures and loss of diagnostic accuracy not only in patients infected with HBV, but also in those with HDV. Therefore, it is advisable and of great significance to adequate the currently available commercial immunoassays kits used in the diagnosis of hepatitis $\mathrm{B}$ for the detection of individuals harboring such emerging viruses, and to reevaluate the HDV diagnostic algorithms.

Due to the high incidence of nucleos(t)ide analogues-resistance associated vaccine-escape HBV mutants among HIV-co infected patients undergoing dual ART, hepatitis B treatment options that aim to reduce the risk of HBV mutations from emerging should be seriously considered, not only from clinical but also from public health perspectives. From a Public Health point of view, it is important to understand the distinct traits of these S-escape mutants in relation to their prevalence and heterogeneity since this knowledge could be of great help for improving diagnostic assays, designing new vaccines, preventing HBIG therapy failure, and reevaluating the hepatitis B treatment options among HIV-co infected patients.

\section{Acknowledgements}

This work was partially supported by the University of Buenos Aires (Grant UBACyT 20020130200222BA). A Nusblat and ML Cuestas are members of CONICET.

\section{Conflicts of interest}

None.

\section{References}

1. WHO. Hepatitis B. World Health Organization Fact sheet $N^{\circ} 204.2015$.

2. El-Seraq HB. Hepatocellular carcinoma. $N$ Engl $J$ Med. 2011;365(12):1118-1127.

3. Gish RG, Locarnini S. Genotyping and genomic sequencing in clinical practice. Clin Liver Dis. 2007;11(4):761-795.

4. Komas NP, Vickos U, Hübschen JM, et al. Cross-sectional study of hepatitis B virus infection in rural communities, Central African Republic. BMC Infect Dis. 2013;13:286.

5. Ducancelle A, Abguguen P, Birguel J, et al. High endemicity and low molecular diversity of hepatitis B virus infections in pregnant women in a rural district of North Cameroon. PLoS One. 2013;8(11):e80346.

6. Lee KM, Kim YS, Koy Y, et al. Emergence of vaccine-induced escape mutant of hepatitis B virus with multiple surface gene mutations in a Korean child. J Korean Med Sci. 2001;16(3):359-362.

7. Basuni AA, Butterworth L, Cooksley G, et al. Prevalence of HBsAg mutants and impact of hepatitis B infant immunization in four Pacific Island countries. Vaccine. 2004;22(21-22):2791-2799.
8. Mathet VL, Cuestas ML, Ruiz V, et al. Detection of hepatitis B virus (HBV) genotype E carried -even in the presence of high titters of anti-HBs antibodies-by an Argentinean patient of African descent who had received vaccination against HBV. J Clin Microbiol. 2006;44(9):3435-3439.

9. Lin YM, Jow GM, Mu SC, et al. Naturally occurring hepatitis B virus B-cell and T-cell epitope mutants in hepatitis B vaccinated children. Scientific World Journal. 2013:571875.

10. Lacombe K, Boyd A, Gozlan J, et al. Drug-resistant and immuneescape HBV mutants in HIV-infected hosts. Antivir Ther. 2010;15(3PtB):493-497.

11. Olivero A, Smedile A. Hepatitis delta virus diagnosis. Seminar Liver Dis. 2012;32(3):220-227.

12. Shen T, Yan XM. Hepatitis B virus genetic mutations and evolution in liver diseases. World J Gastroenterol. 2014;20(18):5435-5441.

13. Price PM, Mohamad A, Zelent A, et al. Translational selection in the expression of the hepatitis B virus envelope proteins. DNA. 1988;7(6):417-422.

14. Cuestas ML, Mathet VL, Oubiña JR, et al. Drug delivery systems and liver targeting for the improved pharmacotherapy of the hepatitis B virus (HBV) infection. Pharm Res. 2010;27(7):1184-1202.

15. Bouchard MJ, Schneider RJ. The enigmatic $X$ gene of hepatitis $B$ virus. J Virol. 2004;78(23):12725-12734.

16. Ganem D, Prince AM. Hepatitis B virus infection-natural history and clinical consequences. $N$ Engl J Med. 2004;350(11):1118-1129.

17. Brunetto MR, Rodriguez UA, Bonino F. Hepatitis B virus mutants. Intervirology. 1999;42(2-3):69-80.

18. Pumpens P, Grens E, Nassal M. Molecular epidemiology and immunology of hepatitis B virus infection - an update. Intervirology. 2002;45(4-6):218-232.

19. Osiowy C, Giles E, Tanaka Y, et al. Molecular evolution of hepatitis B virus over 25 years. $J$ Virol. 2006;80(21):10307-10314.

20. López JL, Mathet VL, Oubiña JR, et al. Intrahost evolution of $\mathrm{HBe}$ antigen-negative hepatitis $\mathrm{B}$ virus genomes ascribed to the $\mathrm{F}$ genotype: a longitudinal 3 year retrospective study. J Gen Virol. 2007;88(Pt):86-91.

21. Mathet VL, López JL, Ruiz V, et al. Dynamics of a hepatitis B virus e antigen minus population ascribed to genotype $\mathrm{F}$ during the course of a chronic infection despite the presence of anti-HBs antibodies. Virus Res. 2007;123(1):72-85.

22. Wai CT, Fontana RJ. Clinical significance of hepatitis B virus genotypes, variants and mutants. Clin Liver Dis. 2004;8(2):321-352.

23. Antoni BA, Rodríguez-Crespo I, et al. Site-directed mutagenesis of cysteine residues of hepatitis B surface antigen. Analysis of two single mutants and double mutants. Eur J Biochem. 1994;222(1):121-127.

24. Seddigh-Tonekaboni S, Waters JA, Jeffers S, et al. Effect of variation in the common "a" determinant on the antigenicity of hepatitis B surface antigen. J Med Virol. 2000;60(2):113-121.

25. Mangold CM, Streeck RE. Mutational analysis of the cysteine residues in the hepatitis B virus small envelope protein. $J$ Virol. 1993;67(8):4588-4597.

26. Chen YC, Delbrook K, Dealwis C, et al. Discontinuous epitopes of hepatitis B surface antigen derived from a filamentous phage peptide library. Proc Natl Acad SCI USA. 1996;93(5):1997-2001.

27. Mangold CM, Unckell F, Werr M, et al. Analysis of intermolecular disulfide bonds and free sulfhydryl groups in hepatitis B surface antigen particles. Arch Virol. 1997;142(11):2257-2267.

28. Carman WF. Infections associated with medical intervention: hepatitis viruses and HGV. Br Med Bull. 1998;54(3):731-748. 
29. Weber B. Genetic variability of the $\mathrm{S}$ gene of hepatitis B virus: clinical and diagnostic impact. J Clin Virol. 2005;32(2):102-112.

30. Cuestas ML, Mathet VL, Ruiz V, et al. Unusual naturally occurring humoral and cellular mutated epitopes of hepatitis B virus in a chronically infected argentine patient with anti-HBs antibodies. J Clin Microbiol. 2006;44(6):2191-2198.

31. de Franchis R, Hadengue A, Lau G, et al. EASL International Consensus Conference on Hepatitis B. 13-14 September, 2002 Geneva, Switzerland. Consensus statement (long version). J Hepatol. 2003;39Suppl1:S3-25.

32. Dindoost P, Jazayeri SM, Karimzadeh H, et al. HBsAg variants: common escape issues. Jundishapur J Microbiol. 2012;5(4):521-527.

33. Yamamoto K, Horikita M, Tsuda F, et al. Naturally occurring escape mutants of hepatitis $B$ virus with various mutations in the $\mathrm{S}$ gene in carriers seropositive for antibody to hepatitis B surface antigen. $J$ Virol. 1994;68(4):2671-2676.

34. Mathet VL, Feld M, Espínola L, et al. Hepatitis B virus S gene mutants in a patient with chronic active hepatitis with circulating anti-HBs antibodies. J Med Virol. 2003;69(1):18-26.

35. Hou J, Kaayiannis P, Waters J, et al. A unique insertion in the $\mathrm{S}$ gene of surface antigen-negative hepatitis B virus Chinese carriers. Hepatology. 1995;21(2):273-278.

36. Shields PL, Owsianka A, Carman WF, et al. Selection of hepatitis B surface "escape" mutants during passive immune prophylaxis following liver transplantation: potential impact of genetic changes on polymerase protein function. Gut. 1999;45(2):306-309.

37. Ogura Y, Kurosaki M, Asahina Y, et al. Prevalence and significance of naturally occurring mutations in the surface and polymerase genes of hepatitis B virus. J Infect Dis. 1999;180(5):1444-1451.

38. Kaymakoglu S, Baran B, Onel D, et al. Acute hepatitis B due to immuneescape mutations in a naturally immune patient. Acta Gastroenterol Belg. 2014;77(2):262-265.

39. Zanetti AR, Tanzi E, Manzillo G, et al. Hepatitis B variant in Europe. Lancet. 1988;2(8620):1132-1133.

40. Zuckerman JN, Zuckerman AJ. Mutations of the surface protein of hepatitis B virus. Antiviral Res. 2003;60(2):75-78.

41. Kreutz C. Molecular, immunological and clinical properties of mutated hepatitis B viruses. J Cell Mol Med. 2002;6(1):113-143.

42. Coleman PF. Surveillance for hepatitis B surface antigen mutants. J Med Virol. 2006;78(S1):56-58.

43. Weinberger KM, Bauer T, Böhm S, et al. High genetic variability of the group -specific a-determinant of hepatitis $B$ virus surface antigen $8 \mathrm{HBsAg}$ ) and the corresponding fragment of the viral polymerase in chronic virus carriers lacking detectable HBsAg in serum. J Gen Virol. 2000;81(Pt5):1165-1174.

44. Hsu HY, Chang MH, Ni YH, et al. Long-term follow-up of children with postnatal immunoprophylaxis failure who were infected with hepatitis B virus surface antigen gene mutants. J Infect Dis. 2013;207(7):1047-1057.

45. Ghany MG, Ayola B, Villamil FG, et al. Hepatitis B virus S mutants in liver transplant recipients who were reinfected despite hepatitis B immune globulin prophylaxis. Hepatology. 1998;27(1):213-222.

46. Ma Q, Wang Y. Comprehensive analysis of the prevalence of hepatitis $\mathrm{B}$ virus escape mutations in the major hydrophilic region of surface antigen. J Med Virol. 2012;84(2):198-206.

47. Kamat SP, Mehta PR, Paranjpe SM, et al. Hepatitis B virus (HBV) infection in liver disease patients in Mumbai, India with special reference to hepatitis B surface antigen (HBsAg) mutant detection. $J$ Clin Diagn Res. 2014;8(3):19-21.

48. Sticchi L, Caligiuri P, Cacciani R, et al. Epidemiology of HBV S-gene mutants in the Liguria Region, Italy: implications for surveillance and detection of new escape variants. Hum Vaccin Immunother. 2013;9(3):568-571.

49. Oon CJ, Chen WN. Current aspects of hepatitis B surface antigen mutants in Singapore. J Viral Hepat. 1998;5Suppl2:17-23.

50. He C, Nomura F, Itoga S, et al. Prevalence of vaccine-induced escape mutants of hepatitis B virus in the adult population in China: a prospective study in 176 restaurant employees. $J$ Gastroenterol Hepatol. 2001;16(12):1373-1377.

51. Bian T, Yan H, Shen L, et al. Change in hepatitis B virus large surface antigen variant prevalence 13 years after the implementation of a universal vaccination program in China. J Virol. 2013;87(22):12196-12206.

52. Hsu HY, Chang MH, Ni YH, et al. No increase in prevalence of hepatitis $\mathrm{B}$ surface antigen mutant in a population of children and adolescents who were fully covered by universal infant immunization. J Infect Dis. 2010;201(8):1192-1200.

53. Jilg W, Schmidt M, Deinhardt F. Prolonged immunity after late booster doses of hepatitis B vaccine. The Journal of Infectious Diseases. 1988;157(6):1267-1269.

54. Gerlich WH. Prophilactic vaccination against hepatitis B: achievements, challenges and perspectives. Med Microbiol Immunol. 2015;204(1):39-55.

55. Lündsorf H, Gurramkonda C, Adnan A, et al. Virus-like particle production with yeast. Ultrastructural and immunocytochemical insights into Pichia pastoris producing high levels of the hepatitis B surface antigen. Microbal cell Factories. 2011;10:48.

56. Cuestas ML, Rivero CW, Minassian ML, et al. Naturally occurring hepatitis B virus (HBV) variants with primary resistance to antiviral therapy and S-mutants with potential primary resistance to adefovir in Argentina. Antiviral Res. 2010;87(1):74-77.

57. Delfino CM, Gentile EA, Castillo AI, et al. Hepatitis B virus and hepatitis D virus in blood dondors from Argentina: circulation of $\mathrm{HBsAg}$ and reverse transcriptase mutants. Arch Virol. 2014;159(5):1109-1117.

58. Romano L, Paladini S, Galli C, et al. Hepatitis B vaccination. Hum Vaccin Immunother. 2015;11(1):53-57.

59. Milazzo L, Ebranati E, Cattaneo D, et al. Recurrence of another hepatitis $B$ virus escape mutants comes back in a patient infected with HIV and low CD4+ count. J Med Virol. 2014;86(1):97-101.

60. Costantini A, Marinelli K, Bagioni G, et al. Molecular analysis of hepatitis $\mathrm{B}$ virus $8 \mathrm{HBV}$ ) in an HIV co-infected patient with reactivation of occult HBV infection following discontinuation of lamivudineincluding antiretroviral therapy. BMC Infect Dis. 2011;11:310.

61. Pal A, Sarkar N, Saha D, et al. High incidence of lamivudine-resistance associated vaccine-escape HBV mutants among HIV-coinfected patients on prolonged antiretroviral therapy. Antivir Ther Epub ahead of print. 2015.

62. Soriano V, de Mendoza C, Peña JM, et al. Advances in treating drugresistant hepatitis B virus in HIV-infected patients. Expert Opin Pharmacother. 2015;16(2):179-1.

63. Lacombe K, Boyd A, Lavocat F, et al. High incidence of treatmentinduced and vaccine-escape hepatitis $\mathrm{B}$ virus mutants among human immunodeficiency virus/hepatitis B-infected patients. Hepatology. 2013;58(3):912-922.

64. Coffin CS, Osiowy C, Myers RP, et al. Virology and clinical sequelae of long term antiviral therapy in a North American cohort of hepatitis $\mathrm{B}$ virus (HBV)/human immunodeficiency virus type $18 \mathrm{HIV}-1)$ coinfected patients. J Clin Virol. 2013;57(2):103-108.

65. Chisari FV, Klopchin K, Moriyama T, et al. Molecular pathogenesis of hepatocellular carcinoma in hepatitis B virus transgenic mice. Cell. 1989;59(6):1145-1156. 
66. Pollicino T, Cacciola I, Saffioti F, et al. Hepatitis B virus PreS/S gene variants: pathobiology and clinical implications. $J$ Hepatol. 2014;61(2):408-417.

67. Asahina Y, Enomoto N, Ogura Y, et al. Sequential changes in full-length genomes of hepatitis B virus accompanying acute exacerbation of chronic hepatitis B. J Hepatol. 1996;25(6):787-794.

68. Torresi J. The virological and clinical significance of mutations in the overlapping envelope and polymerase genes of hepatitis B virus. J Clin Virol. 2002;25(2):97-106.

69. Huang CR, Szecheng JL. Hepatitis D virus infection, replication and cross-talk with the hepatitis B virus. World $J$ Gastroenterol. 2014;20(40):14589-14597.

70. Said ZN. An overview of occult hepatitis B virus infection. World $J$ Gastroenterol. 2011;17(15):1927-1938.

71. Kwak MS, Kim YJ. Occult hepatitis B virus infection. World J Hepatol. 2014;6(12):860-869.

72. Thakur V, Kazim SN, Guptan RC, et al. Transmission of G145R mutants of HBV to an unrelated contact. J Med Virol. 2005;76(1):40-46.

73. Chevrier MC, St-Louis M, Perreault J, et al. Detection and characterization of hepatitis B virus of anti-hepatitis B core antigenreactive blood donors in Quebec with an in-house nucleic acid testing assay. Transfusion. 2007;47(10):1794-1802.

74. Gerlich WH, Bremer L, Saniewski M, et al. Occult hepatitis B virus infection: detection and significance. Dig Dis. 2010;28(1):116-125.

75. Ozaslan E, Purnak T. Is it a case of transfusion-transmitted acute HBV or reactivation of occult hepatitis B virus infection? Transfus Med. 2010;20(4):275.

76. Raimondo G, Caccamo G, Filomia R, et al. Occult HBV infection. Semin Immunopathol. 2013;35(1):39-52.

77. Huang X, Hollinger FB. Occult hepatitis B virus infection and hepatocellular carcinoma: a systemic review. $J$ Viral Hepat. 2014;21(3):153-162.
78. $\mathrm{Mu}$ SC, Lin YM, Jow GM, et al. Occult hepatitis B virus infection in hepatitis B vaccinated children in Taiwan. J Hepatol. 2009;50(2):264-272.

79. Maldonado-Rodriguez A, Cevallos AM, Rojas-Montes O, et al. Occult hepatitis B virus co-infection in human immunodeficiency virus-positive patients: A review of prevalence, diagnosis and clinical significance. World J Hepatol. 2015;7(2):253-260.

80. Delfino CM, Eirin ME, Malan R, et al. HDAg-L variants in covert hepatitis D and HBV occult infection among amerindians of Argentina: new insights. J Clin Virol. 2012;54(3):223-228.

81. Levicnik-Stezinar S. Hepatitis B surface antigen escape mutant in a first time blood donor potentially missed by routine screening assay. Clin Lab. 2004;50(1-2):49-51.

82. Cao GW. Clinical relevance and public health significance of hepatitis B virus genomic variations. World $J$ Gastroenterol. 2009;15(46):5761-5769.

83. Huy TT, Sall AA, Reynes JM, et al. Complete genome sequence and phylogenetic relatedness of hepatitis B virus isolates in Cambodia. Virus Genes. 2008;36(2):299-305.

84. Olinger CM, Lazouskaya NV, Eremin VF, et al. Multiple genotypes and subtypes of hepatitis $\mathrm{B}$ and $\mathrm{C}$ viruses in Belarus: similarities with Russia and western European influences. Clin Microbiol Infect. 2008;14(6):575-581.

85. Kramvis A, Kew MC. Epidemiology of hepatitis B virus in Africa, its genotypes and clinical associations of genotypes. Hepatol Res. 2007;37(s1):S 9-S19.

86. Karthigesu VD, Allison LM, Ferguson M, et al. A hepatitis B virus variant found in the sera of immunized children induces a conformational change in the HBsAg "a" determinant. J Med Virol. 1999;58(4):346-352.

87. Ogata N. Comparison of antibody response to hepatitis B surface antigen among four recipients groups of hepatitis B vaccines that have been approved in Japan: evaluation using passive hemagglutination assay and chemiluminiscent immunoassay. Rinsho Byori. 2009;57(10):954-960. 